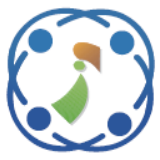

\title{
CT and MRI Image Fusion Based on Discrete Wavelet Transform and Type-2 Fuzzy Logic
}

\author{
Agarwal Ruchi Sanjay ${ }^{1}$, Rajkumar Soundrapandiyan ${ }^{1 *}$, Marimuthu Karuppiah ${ }^{1}$, \\ Rajasekaran Ganapathy ${ }^{2}$ \\ ${ }^{1}$ School of Computer Science and Engineering, VIT University, Vellore, India \\ ${ }^{2}$ School of Advanced Science, VIT University, Vellore, India \\ *Corresponding author's Email: rajkumarsrajkumar@gmail.com
}

\begin{abstract}
Medical imaging requires capturing different aspects of a human body. To capture tissues, cartilages, bones and nerves one needs different sensors and different modalities which results in different images from each modality of the same body part. Image fusion is a process by which one can fuse such images from different modalities in a single image. In this paper, a novel DWT-type2 fuzzy method is proposed to fuse two images (CT and MRI images). In this method, initially, the source images are decomposed into low-level and high-level subbands by discrete wavelet transformation (DWT). As the second step, for fusion, Type-2 fuzzy technique is applied on a low-level subband and average fusion method is applied on the high-level subbands in order to enhance the most prominent features present in CT image and MRI image. Finally, the fused low-level subband and highlevel subbands are reconstructed to form the final fused image using inverse-DWT. To test the proposed fusion method, the experiment is carried out on the MRI-T2 image, functional MRI image and CT image. The fused images have been subjectively and objectively evaluated based on certain evaluation measures and the obtained results have been presented in the paper. From the results, one can observe that the proposed method provides an improvement over other primitive fusion methods.
\end{abstract}

Keywords: Image fusion, Medical image fusion, DWT, Type-2 fuzzy, measures.

\section{Introduction}

Image fusion is an essential step in medical imaging these days. Image fusion is a process by which two images are fused together to obtain a single image. Images with different focused regions, images from different modalities or images taken in different times have been fused together to give enhanced results. In medical field, different multimodal images such as Computed Tomography (CT), Magnetic Resonance Image (MRI), Positron Emission Tomography (PET) and Single Photon Emission Computed Tomography (SPECT) are used to study different aspects of human body part [1]. These images differ with respect to the sensors employed and the exposure to the object. For example, CT image uses X-rays to form a cross- sectional image of the body part in consideration. MRI images can be used to detect the presence of fat, water and other fluids in the body depending upon the radiations used. It works on the principal that certain elements align themselves in presence of an external field. Functional MRI images depict the response of brain to an external stimuli. It detects changes in the blood flow. With change in time, the images produced may differ. These images cannot give a clear picture needed for disease diagnosis, treatment process etc. Thus, for efficient diagnosis, one needs different multimodal image information in a single image. This can be achieved with image fusion techniques.

To this point, in research, many fusion techniques were proposed by the researchers for medical images. These fusion techniques are categorized into pixel, region and decision levels [2]. 
The pixel level fusion is most popular for medical image fusion when compared to region and decision levels. The pixel level fusion is simple and can provide the fusion image without any artifacts. The pixel level fusion techniques are classified into spatial fusion methods and transform fusion methods. The spatial fusion methods include the average method, minimum method, maximum method, contrast pyramid, principal component analysis (PCA) method, laplacian pyramid and so on $[3,4]$. These methods are directly applied on the image pixels and these reduce the signal-to-noise ratio (SNR) in the resultant images but introduce spatial and spectral distortion in the fused image. To resolve this problem, transform-based fusion methods are used. The transform based fusion methods include decomposition of image by stationary wavelet transform (SWT), discrete wavelet transform (DWT), lifting wavelet transform (LWT), redundancy discrete wavelet transform (RDWT), dual-tree complex wavelet transform (DTDWT) and so on [5,6]. These methods have unique drawbacks but all of them share some common drawbacks such as introduced additive noise in fused image.

To address the above issues, a novel approach with DWT-type 2 fuzzy logic is proposed in this paper. In the proposed method, source images are decomposed into low-level subband, high-level subbands using DWT. Next, low-level sub-images are fused using type-2 fuzzy fusion rule and highlevel sub-images are fused using average fusion rule. Finally, inverse DWT is applied on the fused components to obtain the fused image. The advantages of the proposed DWT-type2 fuzzy method can be stated as 1) Adaptive calculation of the required parameters, 2) No need of prior information about source images, 3) The increased contribution of the source image pixels on the fusion process, and 4) improved visibility of the tumor regions.

The rest of the paper is organized as follows: Section-2 elaborates the proposed method, Section-3 discusses the performance evaluation measures, Section-4 briefly describes the experimental results and performance analysis, Conclusion and future work are summarized at the end.

\section{Proposed methodology}

The proposed DWT-type2 fuzzy method consists of three steps: decomposition, fusion and reconstruction. The block diagram of the proposed DWT-type2 fuzzy method is shown in Fig. 1.

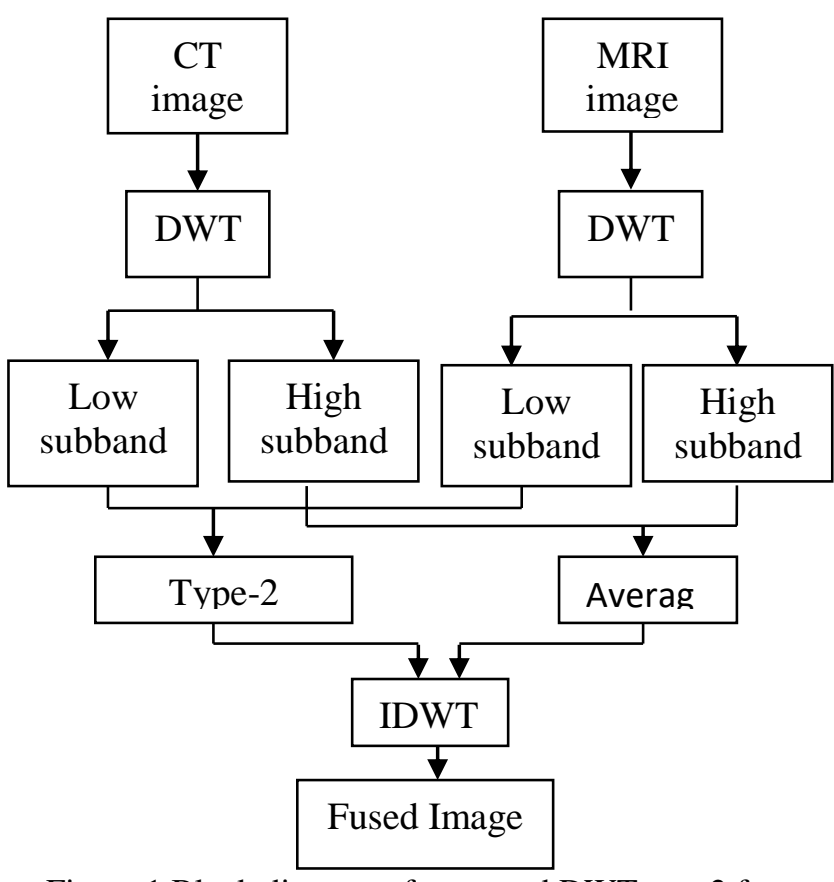

Figure.1 Block diagram of proposed DWT-type2 fuzzy method

The steps involved in the proposed method are as follows:

1. Input two images. One CT image $\left(I_{I}\right)$ and another MRI image $\left(I_{2}\right)$.

2. Decompose both the images using discrete wavelet transformation.

3. Four images will be obtained; approximate sub-image, Horizontal frequency subband, Vertical frequency sub band and Diagonal frequency subband.

4. Perform type-2 fuzzy on the low-frequency subbands that is the fusion of the approximate sub-images.

5. Apply averaging fusion rule on the highfrequency subbands.

6. Apply inverse discrete wavelet transform on the images to obtain a reconstructed fused image.

\subsection{Decomposition}

In the proposed method, a two-level DWT decomposition technique is employed to decompose the input images. In discrete wavelet Transformation wavelets are discretely sampled and hence have knowledge of both location and frequency as opposed to the Fourier transformation. DWT decomposes the image into a low-level subband and high-level subbands. This is the first level of decomposition. The low-level subband is decomposed for the second time at second level to produce another set of low-level, high-level subbands. The four components finally selected for 
the fusion of the images are the approximate subband, Horizontal detail subband, vertical detail subband and diagonal detail subband. The subbands are obtained by passing the image through a series of filters. The filters are defined as the orthogonal discrete function. The property of this transform decomposes the images into an orthogonal set of sub-images. The decompose procedure is defined as

$$
\begin{aligned}
& {\left[c A_{1}, c H_{1}, c V_{1}, c D_{1}\right]=d w t 2\left(I_{1}\right)} \\
& {\left[c A_{2}, c H_{2}, c V_{2}, c D_{2}\right]=d w t 2\left(I_{2}\right)}
\end{aligned}
$$

Where $I_{l}$ and $I_{2}$ are source images, $c A_{l}, c H_{l}, c V_{l}$, $c D_{1}$ and $c A_{2}, c H_{2}, c V_{2}, c D_{2}$ are decomposed coefficients of $I_{1}$ and $I_{2}$ images respectively. Next, the obtained high-frequency and low-frequency subbands of the two images are fused using a fusion algorithm.

\subsection{Fusion}

\subsubsection{Fusion of Low-frequency Sub-images}

Fuzzy sets are used in mathematics to solve the uncertainty problem. The element in the sets is redefined on the basis of a membership function. However, as these functions depend highly on intuition are not flexible and hence the uncertainty problem is not addressed properly. In order to reduce this fuzziness, the membership function is bounded by another fuzzy set with a membership function which forms the second level of the fuzzy technique. This definition of a membership function on a second level is termed as type-2 fuzzy [7]. To reduce the complexity involved in type-2 fuzzy technique, a variable alpha $(\alpha)$ is chosen to define the lower and upper bound of the function.

The low-level subbands obtained after decomposition of the source images are classified into corresponding fuzzy sets based on a defined membership function [8]. These fuzzy sets are then evaluated for the maximum fuzzy entropy which are the best suited coefficients for the fusion process of the subbands. The degree of fuzziness of the subimages can be easily determined using the fuzzy entropy of the image. Hence, the determination of the subbands to be the suitable coefficients of the image fusion frame is made by measuring the degree of fuzziness of each image.

Referring to interval type- 2 fuzzy sets, we define membership function on each low-level frequency sub-bands as given below.

$$
f(i, j)=\frac{1}{1+\left|\frac{L(i, j)-k}{c}\right|^{2}}
$$

Where $k$ is the mean of the approximate image, $c$ is the minimum frequency value of the approximate image, $L$ is the approximate sub-bands of the images $\left(c A_{1}, c A_{2}\right), f$ is the first level fuzzy set.

After the membership function is defined and obtained, the lower and upper bounds of the level-2 membership is obtained based on the variable alpha as chosen.

$$
\begin{aligned}
& f_{\text {Ln }}(x, y)=f(i, j)^{\alpha} \\
& f_{H n}(x, y)=f(i, j)^{\frac{1}{\alpha}}
\end{aligned}
$$

Where $n\left(=c A_{1}, c A_{2}\right)$ denotes the sub-bands, $\alpha$ is the chosen arbitrary value.

According to the information theory, larger the fuzzy entropy a fuzzy set has, more information it contains. Hence, after defining the membership functions, entropy of these fuzzy sets is calculated based on the following formula.

$$
E_{n}=\left(f_{H n}-f_{L n}\right)+\frac{\min \left(f_{L n}, 1-f_{H n}\right)}{\max \left(f_{L n}, 1-f_{H n}\right)}
$$

Based on the entropy, corresponding values of the subbands are chosen to be present in the final image.

$$
c A_{3}=\left\{\begin{array}{l}
c A_{1}, \text { if } E_{1} \geq E_{2} \\
c A_{2}, \text { otherwise }
\end{array}\right.
$$

Where $E_{1}, E_{2}$ are the entropy of the approximate subbands of the images $I_{l}$ and $I_{2}$ respectively.

\subsubsection{Fusion of high-frequency sub-images}

The High-frequency subbands are fused using the averaging filter. Each detailed subbands of the CT image are fused along with its corresponding detailed MRI image. Hence, three fused sub-images are obtained after the process.

$$
\begin{aligned}
& c H_{3}=\text { mean }\left(c H_{1}+c H_{2}\right) \\
& c V_{3}=\operatorname{mean}\left(c V_{1}+c V_{2}\right) \\
& c D_{3}=\operatorname{mean}\left(c D_{1}+c D_{2}\right)
\end{aligned}
$$




\subsection{Reconstruction}

The four sub-images are fused according to the fusion algorithms. Inverse transformation is applied on the obtained four sub-images. Reconstruction is the inverse process of up-sampling of images. A rescaling filter is applied to the approximate subband and wavelet filter is used for the highfrequency subbands. The result of the reconstruction is the final image.

$$
F=i d w t 2\left(c A_{3}, c H_{3}, c V_{3}, c D_{3}\right)
$$

Where $F$ is the Final Fused Image.

\section{Performance evaluation measures}

In order to evaluate the performance of the fused image, subjective and objective measures are used. The subjective evaluation relates with the visual perception. Objective analysis of the fused image is done using various measures such as Image Quality Index (IQI), Mean Absolute Error (MSE), Peak Signal to Noise Ratio (PSNR), Normalized Cross Correlation (NCC) and Structural Similarity Index (SSIM). The measures are as follows [9]:

Let us consider a source image $I(i, j)$ and the fused image $F(i, j)$ of size $P$ x $Q$.

\subsection{Image quality index}

UIQI defines image distortion by a combination of three factors, namely contrast distortion, loss of correlation and luminance distortion [10,17]. The quality index is defined as

$$
U I Q I=\frac{\left(4 \times \sigma_{I F}\right)\left(\mu_{I}+\mu_{F}\right)}{\left(\mu_{I}^{2}+\mu_{F}^{2}\right)\left(\sigma_{I}^{2}+\sigma_{F}^{2}\right)}
$$

Where $\sigma_{I F}$ is the covariance of $I F, \mu_{F}$ denotes the average intensities of image $F, \mu_{I}$ denotes the average intensities of the image $I, \sigma_{F}^{2}$ denotes the variance of image $F$ and $\sigma_{I}^{2}$ denotes the variance of image $I$. The UIQI index value varies from -1 to 1 . Once again, the value 1 indicates the identical characteristics of the two images.

\subsection{Mean absolute error}

MAE measure is used to find the proximity between the fused and the source image [10]. The MAE can be defined as

$$
M A E=\frac{1}{P \times Q} \sum_{i=1}^{P} \sum_{j=1}^{Q}|F(i, j)-I(i, j)|
$$

Lower value indicates greater similarity between the original image and the fused image.

\subsection{Peak signal to noise ratio}

PSNR is used to assess the improvement in the quality of the fused image $[11,12]$ and it is defined as given below.

$$
\begin{aligned}
& M S E=1 / P Q \sum_{i=0}^{P-1} \sum_{j=0}^{Q-1}[F(i, j)-I(i, j)]^{2} \\
& P S N R=10 \log _{10}\left(M A X^{2} / M S E\right)
\end{aligned}
$$

Where $M S E$ is the mean square error value of the image and $M A X$ is the maximum value of an image. A higher PSNR value indicates a better quality of the fused image.

\subsection{Normalized cross correlation}

NCC determines the degree of similarity between the two images and it is defined as [13]

$$
N C C=\frac{\sum_{i=1}^{P} \sum_{j=1}^{Q} F(i, j) \times I(i, j)}{\sqrt{\sum_{i=1}^{P} \sum_{j=1}^{Q} F(i, j)^{2}} \sqrt{\sum_{i=1}^{P} \sum_{j=1}^{Q} I(i, j)^{2}}}
$$

The correlation coefficient range varies from -1 to +1 . A -1 indicates negative relationship and +1 indicates positive relationship.

\subsection{Structural similarity index}

SSIM is a measure used to measure the similarity between the fused and source image [14]. The SSIM can be defined as

$$
S S I M=\frac{\left(\left(2 \mu_{F} \mu_{I}+C_{1}\right) \times\left(2 \sigma_{F I}+C_{2}\right)\right)}{\left(\left(\mu_{F}^{2}+\mu_{I}^{2}+C_{1}\right) \times\left(\sigma_{F}^{2}+\sigma_{I}^{2}+C_{2}\right)\right)}
$$

where $\mu_{F}$ denotes the average intensity of image $F$, $\mu_{I}$ denotes the average intensity of image $I, \sigma_{F}$ denotes the variance of image $F, \sigma_{I}$ denotes the variance of image $I, \sigma_{F}$ gives the covariance of $F$ and $I$, and $C_{I}$ and $C_{2}$ are constants. The SSIM index value varies from -1 to 1 . The value 1 signifies that the two images are same in all views. 
Table 1. Comparative Analysis of IQI Values for Different Fusion Methods

\begin{tabular}{|c|c|c|c|c|c|c|c|}
\hline & Dataset1 & Dataset2 & Dataset3 & Dataset4 & Dataset5 & Dataset6 & Ranking \\
\hline Average & 0.5812 & 0.5555 & 0.5438 & 0.5357 & 0.5588 & 0.5761 & 4 \\
\hline Max & 0.7173 & 0.6488 & 0.6815 & 0.6580 & 0.6676 & 0.6849 & 2 \\
\hline Min & 0.2195 & 0.2348 & 0.1929 & 0.2190 & 0.2310 & 0.2410 & 10 \\
\hline Contrast pyramid & 0.2756 & 0.2467 & 0.2236 & 0.2408 & 0.2687 & 0.2965 & 9 \\
\hline Laplacian pyramid & 0.4808 & 0.4692 & 0.4479 & 0.4098 & 0.4670 & 0.4735 & 7 \\
\hline Morphological & 0.5925 & 0.5938 & 0.5637 & 0.5117 & 0.5623 & 0.5899 & 3 \\
\hline PCA & 0.4914 & 0.2770 & 0.3666 & 0.3750 & 0.4103 & 0.4833 & 8 \\
\hline Ratio & 0.5435 & 0.5295 & 0.5088 & 0.5004 & 0.5217 & 0.5477 & 6 \\
\hline DWT & 0.5701 & 0.5453 & 0.5250 & 0.4984 & 0.5504 & 0.5626 & 5 \\
\hline DWT+type2 fuzzy & 0.8481 & 0.8534 & 0.8341 & 0.8362 & 0.8417 & 0.8365 & 1 \\
\hline
\end{tabular}

Table 2. Comparative Analysis of MAE Values for Different Fusion Methods

\begin{tabular}{|c|c|c|c|c|c|c|c|}
\hline & Dataset1 & Dataset2 & Dataset3 & Dataset4 & Dataset5 & Dataset6 & Ranking \\
\hline Average & 0.0767 & 0.1092 & 0.0920 & 0.0903 & 0.0861 & 0.0764 & 3 \\
\hline Max & 0.0767 & 0.1292 & 0.0948 & 0.1000 & 0.0947 & 0.0814 & 4 \\
\hline Min & 0.0805 & 0.0927 & 0.0930 & 0.0844 & 0.0812 & 0.0750 & 2 \\
\hline Contrast pyramid & 0.1023 & 0.1393 & 0.1245 & 0.1170 & 0.1117 & 0.0966 & 9 \\
\hline Laplacian pyramid & 0.0952 & 0.1307 & 0.1153 & 0.1182 & 0.1069 & 0.0910 & 8 \\
\hline Morphological & 0.0877 & 0.1192 & 0.1097 & 0.1135 & 0.1029 & 0.0882 & 6 \\
\hline PCA & 0.0875 & 0.1540 & 0.1167 & 0.1124 & 0.1052 & 0.0858 & 7 \\
\hline Ratio & 0.1203 & 0.1663 & 0.1458 & 0.1455 & 0.1373 & 0.1232 & 10 \\
\hline DWT & 0.0841 & 0.1236 & 0.1050 & 0.1059 & 0.0959 & 0.0799 & 5 \\
\hline DWT+type2 fuzzy & 0.0321 & 0.0339 & 0.0329 & 0.0323 & 0.0325 & 0.0324 & 1 \\
\hline
\end{tabular}

Table 3. Comparative Analysis of PSNR Values for Different Fusion Methods

\begin{tabular}{|c|c|c|c|c|c|c|c|}
\hline & Dataset1 & Dataset2 & Dataset3 & Dataset4 & Dataset5 & Dataset6 & Ranking \\
\hline Average & 65.1992 & 63.0353 & 64.0014 & 64.1370 & 64.5130 & 65.0229 & 2 \\
\hline Max & 61.9037 & 59.1053 & 60.5769 & 60.3860 & 60.8278 & 61.7587 & 9 \\
\hline Min & 62.5332 & 61.2483 & 61.4889 & 62.0707 & 62.3540 & 62.3243 & 7 \\
\hline Contrast pyramid & 62.3787 & 60.488 & 61.0888 & 61.5919 & 61.9570 & 62.4849 & 8 \\
\hline Laplacian pyramid & 63.1009 & 61.2877 & 61.7193 & 61.4946 & 62.4152 & 63.0824 & 5 \\
\hline Morphological & 63.1321 & 61.4744 & 61.4206 & 61.3376 & 62.1062 & 62.7559 & 6 \\
\hline PCA & 64.1017 & 60.0765 & 61.9790 & 62.2710 & 62.8076 & 64.0457 & 4 \\
\hline Ratio & 60.6252 & 58.8302 & 59.4417 & 59.5788 & 60.0241 & 60.6243 & 10 \\
\hline DWT & 64.5931 & 62.0662 & 62.9434 & 62.7618 & 63.7158 & 64.5815 & 3 \\
\hline DWT+type2 fuzzy & 71.0152 & 70.7934 & 70.8951 & 70.9356 & 70.9618 & 70.8865 & 1 \\
\hline
\end{tabular}

Table 4. Comparative Analysis of NCC Values for Different Fusion Methods

\begin{tabular}{|c|c|c|c|c|c|c|c|}
\hline & Dataset1 & Dataset2 & Dataset3 & Dataset4 & Dataset5 & Dataset6 & ranking \\
\hline Average & 0.9101 & 0.8403 & 0.8591 & 0.8783 & 0.8930 & 0.9049 & 3 \\
\hline Max & 0.8879 & 0.8367 & 0.8451 & 0.8532 & 0.8706 & 0.8854 & 6 \\
\hline Min & 0.8309 & 0.7041 & 0.7280 & 0.7935 & 0.8141 & 0.8186 & 10 \\
\hline Contrast pyramid & 0.8481 & 0.7462 & 0.7624 & 0.8082 & 0.8308 & 0.8512 & 9 \\
\hline Laplacian pyramid & 0.8911 & 0.8382 & 0.8385 & 0.8412 & 0.8744 & 0.8918 & 5 \\
\hline Morphological & 0.9000 & 0.8476 & 0.8403 & 0.8547 & 0.8783 & 0.8966 & 4 \\
\hline PCA & 0.8845 & 0.7207 & 0.7809 & 0.8183 & 0.8455 & 0.8812 & 8 \\
\hline Ratio & 0.8687 & 0.8328 & 0.8329 & 0.8472 & 0.8616 & 0.8690 & 7 \\
\hline DWT & 0.9127 & 0.8475 & 0.8574 & 0.8663 & 0.8964 & 0.9112 & 2 \\
\hline DWT+type2 fuzzy & 0.9773 & 0.9718 & 0.9724 & 0.9750 & 0.9760 & 0.9763 & 1 \\
\hline
\end{tabular}


Table 5. Comparative Analysis of SSIM Values for Different Fusion Methods

\begin{tabular}{|c|c|c|c|c|c|c|c|}
\hline & Dataset1 & Dataset2 & Dataset3 & Dataset4 & Dataset5 & Dataset6 & Ranking \\
\hline Average & 0.9983 & 0.9967 & 0.9975 & 0.9975 & 0.9978 & 0.9982 & 2 \\
\hline Max & 0.9952 & 0.9904 & 0.9933 & 0.9929 & 0.9937 & 0.9950 & 9 \\
\hline Min & 0.9972 & 0.9958 & 0.9959 & 0.9967 & 0.9970 & 0.9971 & 3 \\
\hline Contrast Pyramid & 0.9974 & 0.9953 & 0.9961 & 0.9966 & 0.9969 & 0.9975 & 4 \\
\hline Laplacian pyramid & 0.9967 & 0.9951 & 0.9954 & 0.9950 & 0.9960 & 0.9966 & 7 \\
\hline Morphological & 0.9969 & 0.9953 & 0.9952 & 0.9948 & 0.9957 & 0.9965 & 8 \\
\hline PCA & 0.9978 & 0.9935 & 0.9960 & 0.9962 & 0.9967 & 0.9977 & 6 \\
\hline Ratio & 0.9943 & 0.9905 & 0.9920 & 0.9922 & 0.9931 & 0.9942 & 10 \\
\hline DWT & 0.9977 & 0.9958 & 0.9966 & 0.9963 & 0.9970 & 0.9976 & 5 \\
\hline DWT+type2 fuzzy & 0.9999 & 0.9999 & 0.9999 & 0.9999 & 0.9999 & 0.9999 & 1 \\
\hline
\end{tabular}

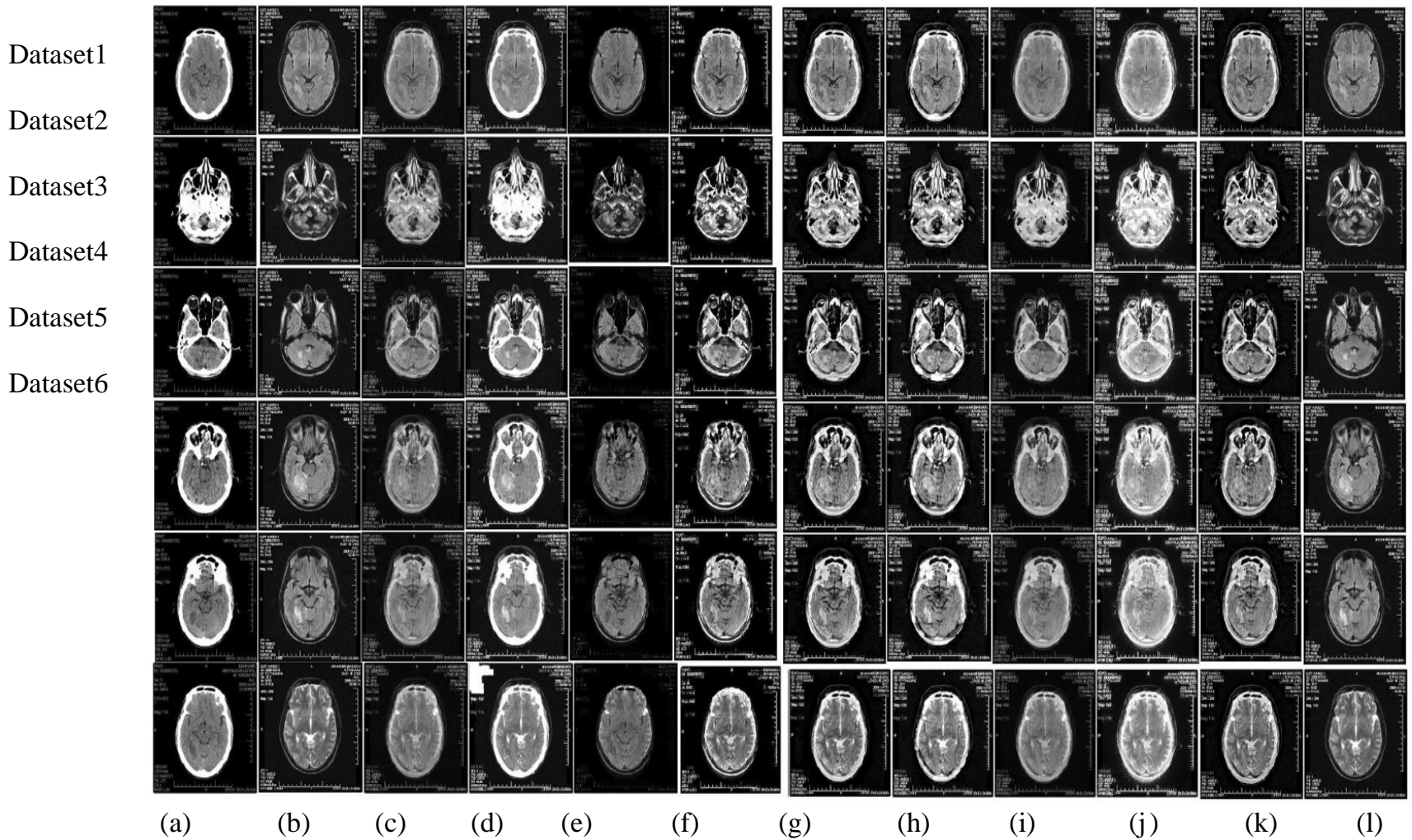

Figure.2 Subjective comparison of the fusion results: (a) CT image, (b) MRI images, (c) The result of average method, (d)The result of maximum method, (e) The result of minimum method, (f) The result of contrast pyramid, (g) The result of laplacian pyramid, (h)The result of morphological method, (i) The PCA method, (j) The result of ratio method, (k) The result of DWT, and (1) The result of proposed DWT-type2 fuzzy

\section{Experimental results and performance analysis}

In this section, the experimental results and evaluation of the performance of the proposed fusion method to prove its efficiency are shown.

\subsection{Input}

The CT, MRI-T2 and MRI-FLAIR brain images are used as input images for experimental purpose. These images of same patient are taken from different modalities. For analysis, six brain datasets are used. Each dataset contains one CT and one MRI image. These datasets are classified into two classes. Class 1 consists of dataset 1 to 5 which contains CT and MRI-T2 images, Class 2 consists of dataset 6 which contains MRI-FLAIR and CT images. The size of each image is $256 * 256$.

\subsection{Subjective analysis}

Subjective analysis is carried out on Class 1 and Class 2 source images are CT and MRI-T2 time images and CT and MRI-FLAIR time images as shown in Fig. 2. In Fig. 2, images are ordered column wise - First column CT image, second column MRI image, third column to twelfth column 
- fused images (result of different fusion method) arranged as average [3], maximum [3], minimum [2], contrast pyramid [11], laplacian pyramid [12], morphological [2], PCA [12], ratio pyramid [2], DWT [1] and proposed DWT-type2 fuzzy. From the result it is observed that the visibility of tumor region is increased in the image results of proposed DWT-type2 fuzzy when compared with the results of other methods. The proposed method gives better visualization because of the most prominent features are choosen based on the higher of the value of fuzzy entropy to fuse the low subbands coefficients. The entropy gives the texture information of the image and it is important factor for the fusion of images. Hence, the proposed method gives better result than the existing methods.

\subsection{Objective analysis}

Using subjective analysis [16] we cannot judge the complete fusion image. Thus, objective analysis is carried out on fused images with measures mentioned in the previous section performance evaluation measures. For each measure, the result obtained from the proposed DWT-type2 fuzzy method is compared with average, maximum, minimum, contrast pyramid, laplacian pyramid, morphological, PCA, ratio pyramid, DWT fusion methods. The comparative analysis of different measures are tabulated in Tables 1,2,3,4 and 5. For easily analysis, the fusion result for each measure is ranked [15]. A fusion result with the best measure value is given rank 1 and worst fusion method is ranked 10, as depicted in the last column of the Tables 1 to 5. From the result, one can deduce that the proposed DWT-type2 fuzzy method produces a better result than the other existing fusion methods.

\section{Conclusion and future work}

In this paper, a novel approach is proposed for fusion of images obtained from different modalities based on discrete wavelet transformation and type- 2 fuzzy method. Here, a two-level DWT decomposition is used to extract the low-frequency and high-frequency subbands. This makes computations simple. For fusion of low-level subbands and high-level subbands Type-2 fuzzy logic and average fusion rule are applied respectively. The fusion rule is applied on low-level sub-images to include the most prominent features with the highest degree of certainty. Then, fused images are reconstructed using inverse DWT. Evaluation results of the fused image based on IQI, MSE, PSNR, NCC and SSIM measures shows that the proposed DWT-type-2 fuzzy fusion method provides better results over the existing methods and it is tabulated in Tables 1 to 5. In addition, the proposed DWT-Type-2 Fuzzy Technique provides improved clinical perspective which is useful for disease diagnosis and easy retrieval. In future, we will be working on employing a different decomposition technique for the images.

\section{References}

[1] S. Rajkumar, P. Bardhan, S. K. Akkireddy, \& C. Munshi, "CT and MRI image fusion based on Wavelet Transform and Neuro-Fuzzy concepts with quantitative analysis", In Proc. of International Conf. on Electronics and Communication Systems, Coimbatore, India, pp.1-6, 2014

[2] Z. Wang, D. Ziou, C. Armenakis, D. Li, Q. Li, "A comparative analysis of image fusion methods", IEEE transactions on Geoscience and Remote Sensing, Vol. 43, No.6, pp.13911402, 2005.

[3] K. Sharmila, S. Rajkumar, \& V. Vijayarajan, "Hybrid method for multimodality medical image fusion using Discrete Wavelet Transform and Entropy concepts with quantitative analysis", International Conference on Communications and Signal Processing (ICCSP), melmaruvathur , India, pp.489-493, 2013.

[4] S. Rajkumar, S. Kavitha, "Redundancy Discrete Wavelet Transform and Contourlet Transform for Multimodality Medical Image Fusion with Quantitative Analysis", In Proc. of $3^{\text {rd }}$ International Conf. on Emerging Trends in Engineering and Technology, Goa, India, pp.134-139, 2010.

[5] S.M. Darwish, "Multi-level fuzzy contourletbased image fusion for medical application", IET Image Processing, Vol. 7, No.7, pp.694-700, 2013.

[6] D. Agrawal, and J. Singhai., "Multifocus image fusion using modified pulse coupled neural network for improved image quality", IET image processing, Vol.4, No.6, pp.443-451, 2010.

[7] Y. Yang, Y. que, S. Huang, P. Lin, "Multimodal Sensor Medical Image Fusion Based on Type-2 Fuzzy Logic in NSCT Domain", IEEE Sensors Journal, Vol.16, No.10, pp.3735-3745, 2016.

[8] Y. Yang, S. Tong, S. Huang, P. Lin., "Multifocus image fusion based on NSCT and focused area detection", IEEE Sensors Journal, Vol.15, No.5, pp.2824-2838, 2015. 
[9] R. Hassen, Z. Wang and M.M. Salama., "Objective quality assessment for multiexposure multifocus image fusion", IEEE Transactions on Image Processing, Vol.24, No.9, pp.2712-2724, 2015.

[10] R. Soundrapandiyan, \& C. M. PVSSR, "Perceptual Visualization Enhancement of Infrared Images Using Fuzzy Sets", In Transactions on Computational Science, Voi. 1, No. 1, pp.3-19, 2015.

[11] S. Gupta, S. Rajkumar, V. Vijayarajan, k. Marimuthu, "Quantitative Analysis of various Image Fusion techniques based on various metrics using different Multimodality Medical Images", International Journal on Engineering and Technology, Vol. 5, No.1, pp.133-141, 2013.

[12] S. Rajkumar, R. Haldar, A. Pillai, P. Duta, "A Sugeno fuzzy logic based CT and MRI image fusion technique with quantitative analysis", International Journal of Pharmacy and Technology, Vol.8, No.1, pp.11286-11296, 2016.

[13] S. Rajkumar, S. Ramani, P. Boominathan, "Medical Image Fusionusing Contourlet Transformand Fusion Tool Techniques", International Journal of Pharmacy and Technology, Vol.8, No.2, pp.13553-13563, 2016.

[14] C. Prakash, S. Rajkumar, P.V.S.S.R. Chandramouli, "Medical Image Fusion based on Redundancy DWT and Mamdani type min sum mean-of-max techniques with Quantitative Analysis", In Proc. of International Conf. on Recent Advances in Computing and Software Systems, Chennai, India, pp.54-59, 2012.

[15] D.P. Bavirisetti and R. Dhuli. "Multi-focus image fusion using maximum symmetric surround saliency detection", ELCVIA Electronic Letters on Computer Vision and Image Analysis, Vol.4, No.2, pp.58-73., 2016.

[16] R. Soundrapandiyan, and P.V.S.S.R. Chandra Mouli, "A novel and robust rotation and scale invariant structuring elements based descriptor for pedestrian classification in infrared images", Infrared Physics \& Technology, Vol.78, pp.1323, 2016.

[17] R. Soundrapandiyan, and P.V.S.S.R. Chandra Mouli, "Adaptive pedestrian detection in infrared images using fuzzy enhancement and top-hat transform", International Journal of Computational Vision and Robotics, Vol.7, No.1/2, pp.49-67, 2017. 